OPEN ACCESS

Edited by:

Laura Ballerini,

Scuola Internazionale di Studi

Superiori Avanzati (SISSA), Italy

Reviewed by:

Marco Atzori,

Universidad Autónoma de San Luis

Potosí, Mexico

Preeti Sareen,

Yale University, United States

*Correspondence:

María C. Serrano

mc.terradas@csic.es

Received: 14 April 2017 Accepted: 14 September 2017 Published: 27 September 2017

Citation:

Domínguez-Bajo A,

González-Mayorga A,

López-Dolado E and Serrano MC

(2017) Graphene-Derived Materials Interfacing the Spinal Cord:

Outstanding in Vitro and in Vivo Findings.

Front. Syst. Neurosci. 11:71. doi: 10.3389/fnsys.2017.00071

\section{Graphene-Derived Materials Interfacing the Spinal Cord: Outstanding in Vitro and in Vivo Findings}

\author{
Ana Domínguez-Bajo ${ }^{1}$, Ankor González-Mayorga ${ }^{1}$, Elisa López-Dolado ${ }^{1,2}$ \\ and María C. Serrano $2,3,4$ *
}

\begin{abstract}
'Laboratory of Interfaces for Neural Repair, Hospital Nacional de Parapléjicos, Servicio de Salud de Castilla-La Mancha, Toledo, Spain, ${ }^{2}$ Research Unit of "Design and development of biomaterials for neural regeneration", Hospital Nacional de Parapléjicos, Servicio de Salud de Castilla-La Mancha, Joint Research Unit with CSIC, Toledo, Spain, ${ }^{3}$ Laboratory of Materials for Health, Instituto de Ciencia de Materiales de Madrid, Consejo Superior de Investigaciones Cientificas, Madrid, Spain, ${ }^{4}$ Materials Science Factory, Instituto de Ciencia de Materiales de Madrid, Consejo Superior de Investigaciones Cientificas, Madrid, Spain
\end{abstract}

The attractiveness of graphene-derived materials (GDMs) for neural applications has fueled their exploration as components of biomaterial interfaces contacting the brain and the spinal cord. In the last years, an increasing body of work has been published on the ability of these materials to create biocompatible and biofunctional substrates able to promote the growth and activity of neural cells in vitro and positively interact with neural tissues when implanted in vivo. Encouraging results in the central nervous tissue might impulse the study of GDMs towards preclinical arena. In this mini-review article, we revise the most relevant literature on the interaction of GDMs with the spinal cord. Studies involving the implantation of these materials in vivo in the injured spinal cord are first discussed, followed by models with spinal cord slides ex vivo and a final description of selected results with neural cells in vitro. A closing debate of the major conclusions of these results is presented to boost the investigation of GDMs in the field.

Keywords: graphene, spinal cord injuries, scaffold, toxicity, neurons, in vivo models

\section{INTRODUCTION}

The outstanding physico-chemical properties of carbon-based materials including carbon nanotubes (CNTs), carbon nanofibers and graphene are encouraging their exploration as attractive materials to interface the damaged neural tissue as part of novel platforms with potential utility in neural repair (Tavangarian and Li, 2012). One of the main properties of these materials is their ability to adsorb molecules. This fact brings high versatility for biological applications due to the possibility of modifying their surface characteristics to induce and modulate specific cell and tissue responses. Specifically, graphene displays a large surface area enhancing adsorptive features (Li et al., 2008). Graphene oxide (GO) presents a higher ability to adsorb molecules as a consequence of the presence of oxygen-containing chemical groups that serve attracting adhesive moieties, cell media components and therapeutic drugs (Yang et al., 2008). Moreover, GO is more hydrophilic than pristine graphene, thus increasing dispersibility and diminishing aggregation. An additional feature that make graphene-derived materials (GDMs) attractive for interfacing the injured central nervous tissue is their capacity to cross the blood-brain and blood-spinal cord barriers by using specific functionalization protocols (Yang et al., 2015). Other remarkable properties, such as their mechanical behavior (Lee et al., 2008), allow the preparation of flexible 3D structures 
which are mechanically compliant with neural tissues. Finally, their superior charge carrier mobility (Soldano et al., 2010) is of interest for electrical stimulation and recording in neural tissues and cells.

This mini-review article focuses mainly on the most relevant and recent publications to date on the exploration of GDMs interfacing the spinal cord, including in vivo, ex vivo and in vitro models. Outstanding work of GDMs implanted in the brain is also discussed. Major findings discussed in this mini-review article are summarized in Table 1 . Readers are referred to excellent reviews in the topic for further details (Fattahi et al., 2014; Fraczek-Szczypta, 2014; Nakanishi et al., 2014; John et al., 2015).

\section{SPINAL CORD INJURY AND ITS PHYSIOPATHOLOGY}

Human spinal cord injuries (SCIs) affect thousands of people worldwide every year, leading devastating consequences such as sensory loss, paralysis and bowel/bladder dysfunctions that cost millions of dollars in medical expenses. According to the World Health Organization, although there is no reliable estimation of global prevalence, estimated annual incidence is $40-80$ cases per million worldwide. Unfortunately, less than 1\% of SCI patients achieve complete neurological recovery by hospital discharge (Spinal Cord Injury (SCI), 2016).

Forthwith after SCI, a rapid activation of glial cells and pericytes occurs, forming a complex healing tissue that is simultaneously beneficial and detrimental for neural repair (Göritz et al., 2011). While in the acute phase macrophages and leukocytes are concentrated in the injury, astrocytes, oligodendrocytes and meningeal cells create a non-permissive biochemical barrier for axonal regrowth at the chronic stage (Rodriguez et al., 2014). Importantly, the early inflammatory responses and the suppression of the astrocytic reactivity increase the extent of damage (Fawcett et al., 2012), a still unknown phenomenon in which the rupture of the bloodspinal cord barrier and the microvascular remodeling in the lesion zone might play key roles (Figley et al., 2014). In this scenario, it is widely accepted that intercommunication between neural and immune cells is crucial to induce reparative responses (Kokaia et al., 2012), with different macrophage populations mediating from neurotoxic to neuroregenerative responses (Kigerl et al., 2009). In the acute phase, the compromise of the immune system is directly related to the ischemic, necrotic and inflammatory tissue damage typical of SCI (Sapru, 2011). During the chronic phase, there are other pathogenic factors responding to the immune disturbance such as dysregulation mediated by dysautonomia (Garstang and Walker, 2011) or gut dysbiosis (Kigerl et al., 2016).

Nowadays, the cure of SCI remains a major challenge for both researchers and clinicians. There are two main hypotheses for the lack of effective therapies: (a) human spinal motor system relies more dependently on supraspinal control than in other mammals (Capaday, 2002); and (b) SCI produces such state of spinal circuitry depression that diminishes the capacity of these neural networks to generate responses (Harkema, 2008). Other adverse associated factors are the rapid development of inhibitory fibroglial scars and the limited intrinsic ability of the central nervous tissue to regenerate (Fawcett and Asher, 1999; Chen et al., 2002). In this context, research efforts on the development of new materials such as GDMs with ability to initiate and/or mediate suitable reparative responses in the injured neural tissue have a remarkable interest for human health care.

\section{GRAPHENE-DERIVED MATERIALS INTERFACING THE SPINAL CORD IN VIVO}

\section{Studies with Focus on Toxicity and/or Neural Repair}

López-Dolado et al. (2015) were first to study the in vivo tissue response of the injured rat spinal cord to the implantation of flexible and porous 3D scaffolds composed of reduced graphene oxide ( $\mathrm{rGO}$ ). These scaffolds were fabricated by using the ice segregation-induced self-assembly (ISISA) technique. The lesion model of choice was a right hemisection of approximately $8 \mathrm{~mm}^{3}$ at the C6 level, rostral to the bulk of triceps brachii motoneurons. This is a suitable model to evaluate therapeutic strategies aimed at promoting neural plasticity and repair. In the main experimental group, rGO scaffolds were placed at the lesion site and covered with a thin gelatin hydrogel film. Animals without injury and those hemisected but not receiving scaffolds served as control groups. In order to study the subacute tissue response to these implants, both locally (at the spinal cord) and systemically (in liver, kidney, lung and spleen), rats were sacrificed at 10 days after surgery. The results revealed that these substrates allowed the formation of a soft interface at the injury site, with no significant differences in the fibroglial scar features with respect to lesions without scaffolds. Due to its porous structure, extracellular matrix molecules (e.g., collagen) and different kinds of cells were able to infiltrate and migrate to the inner parts of the scaffolds contributing to the stabilization of both the scaffold and the lesion site. Colonizing cells were mainly positive for vimentin (indicative of connective tissue cells, glial cells and pericytes, among others) and the $\beta$ receptor of the platelet-derived growth factor (a regulator of blood vessels formation and early hematopoiesis). In addition, pro-regenerative M2 macrophages were present both at the interface tissue-material and within the scaffold, which could be potentially involved in the initiation of neural repair responses. Finally, neural cell populations were preserved at the perilesional areas and no toxic systemic responses were found, thus indicating the positive biocompatibility of these GDMs with both neural and non-neural tissues once implanted in vivo.

Fueled by these encouraging findings, the authors focused on the tissue responses at 30 days after surgery (early chronic state; López-Dolado et al., 2016). In these studies, it was first confirmed that the scaffolds were properly stabilized, as already achieved at 10 days. As expected from the progression of the lesion, enhanced cell and collagen infiltration within 


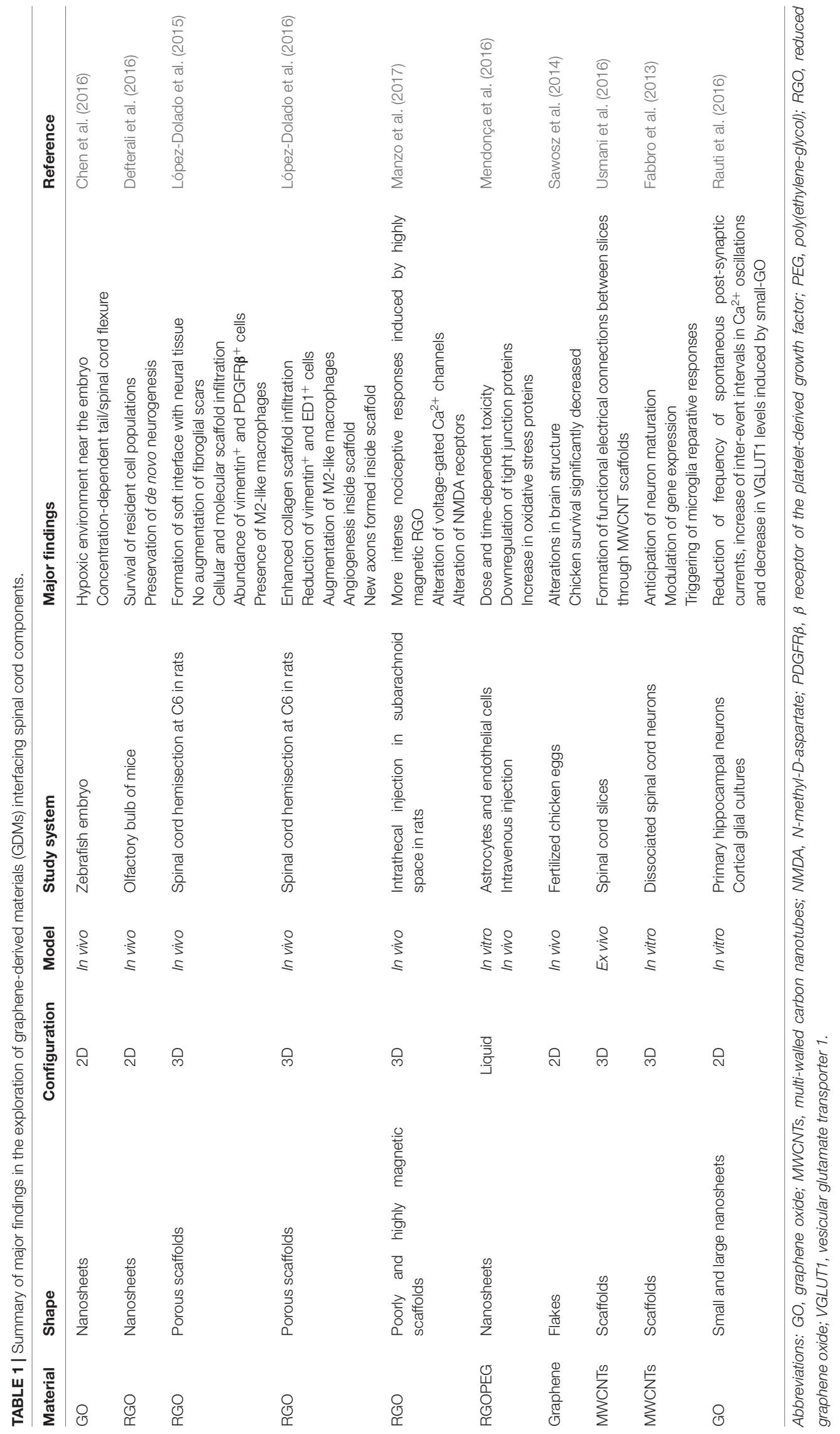


the scaffold was found despite a significant reduction in cells positive for vimentin in comparison to the subacute state. Furthermore, blood vessels, crucial for the initiation of any regenerative processes, increased their size and number over time, both at the interface and, more importantly, inside the graphene substrates. Even though the total amount of macrophages was reduced in comparison with the subacute state, M2 macrophages increased with respect to those with M1-like phenotype (pro-inflammatory). All these changes prompted the formation of a new ambient that allowed the growth of some neuronal axons within the scaffold (not observed at 10 days post-surgery), which were close to functional blood vessels (Figure 1). As found in the subacute state, no evidences of atrophy, inflammation or fibrosis were detected in peripheral organs.

At the brain, Defterali et al. (2016) explored the biocompatibility of $\mathrm{rGO}$ and its influence on neurogenesis in the adult mice olfactory bulb (OB) in vivo. Specifically, a dispersion of rGO nanosheets in phosphate buffer saline $(0.004 \mu \mathrm{g} / \mu \mathrm{L})$ was injected in the OB core. By a simultaneous injection of a retroviral vector expressing the enhanced green fluorescent protein, proliferative cells and their progeny were labeled in the subependymal zone/rostral migratory stream of the $\mathrm{OB}$ core in the presence of rGO. Major findings revealed that rGO had no deleterious effects either on the survival (measured as number of cells and detection of apoptosis) of the resident populations of neurons and astrocytes or on the newly generated neurons (measured as number of cells). By looking at the number and morphology of microglia cells $\left(\mathrm{Iba}^{+}{ }^{+}\right.$), authors evidenced that alterations on the microglial response at the $\mathrm{OB}$ were related to the injection itself rather than the presence of rGO.

Recent studies by Mendonça et al. (2016) focused on the effect of rGO on the blood-brain barrier (BBB) components both in vitro and in vivo. Under in vitro conditions, the exposure to high concentrations of $\mathrm{rGO}(100 \mu \mathrm{g} / \mathrm{mL})$ produced a lower toxicity in both rat astrocytes and endothelial cells than rGO functionalized with poly(ethylene glycol) (PEG), which induced a dose- and time-dependent toxicity. For the in vivo studies, rGO and rGO-PEG were injected intravenously and their toxic effects on BBB integrity analyzed for different times. Both materials caused a notable downregulation of astrocyte markers (GFAP and connexin-43), endothelial tight (occludin) and adherens ( $\beta$-catenin) junctions and basal lamina (laminin) at $3 \mathrm{~h}$ after administration. Interestingly, this effect disappeared after 7 days of exposure to rGO, while in the rGO-PEG group it was permanent and increased over time. The increase in proteins related to the antioxidant system, such as catalase, in the animals treated with $\mathrm{rGO}-\mathrm{PEG}$, accompanied by high levels of intracellular reactive oxygen species, pointed out oxidative stress as one of the main causes of rGO-PEG-mediated damage.

\section{Studies with Focus on Electro-Magnetic Properties of Graphene}

Although more rarely explored, the magnetic properties of GDMs are also attracting attention for their application in neural scenarios. Specifically, magnetic GDMs can generate static magnetic fields (SMFs) that should be taken into account when interfacing the nervous tissue because of their potential influence in cellular intrinsic magnetic fields related to neuronal transmission. As nociception responses depend on this transmission, they could be indirectly influenced by the presence of GDMs. Based on this, Manzo et al. (2017) recently explored the role played by neuronal magnetic fields in nociception by investigating the nociceptive responsiveness to two magnetically-distinct rGO scaffolds (i.e., highly and poorly magnetic). Despite displaying similar chemical features, their respective synthesis processes made them differ in their magnetic behavior. After intrathecal injection in the subarachnoid space of rats, highly magnetic rGO induced more intense nociceptive responses than poorly magnetic one without involving any cytotoxic or inflammatory responses. In vitro experiments also demonstrated that the number of $\mathrm{KCl}$-responsive dorsal root ganglion neurons was greater when treated with highly magnetic rGO. Additional studies showed that the activity of both voltage-gated calcium channels and NMDA receptors was altered in the presence of highly magnetic rGO. The disturbance in the neuronal magnetic field increased nociceptive responsiveness, suggesting a role for the magnetic component of the electromagnetic field in neuronal transmission.

\section{Studies with Focus on Embryogenesis}

Boosted by the tremendous development of GDMs in the industry, Chen et al. (2016) pioneered the investigation of environmental risks associated to GO release to the ecosystem by using a zebrafish embryo model. The results evidenced that GO adhered to and enveloped the chorion, blocking pore canals of its membrane and causing subsequent hypoxia and hatching delay. Moreover, GO was able to penetrate the chorion and enter the embryo by endocytosis. Once inside, it damaged mitochondria and translocated to elements of the circulatory system such as the eye, the heart and the yolk sac, with signs of increased oxidative stress, DNA damage, lipid peroxidation and apoptosis. Adverse effects of GO on heart rate and tail/spinal cord flexure were concentration-dependent, evidencing potential risks for embryogenesis in diverse organs including the central nervous system.

In a different study with fertilized chicken eggs in vivo, graphene toxicity was studied by inoculating different concentrations of pristine graphene (from $50 \mu \mathrm{g} / \mathrm{L}$ to $10,000 \mu \mathrm{g} / \mathrm{L}$ ) dispersed in distilled water (Sawosz et al., 2014). After 19 days of incubation, chicken survival was significantly decreased due to graphene exposure, although neither changes in body and organ weights nor modifications in biochemical indices were found with respect to control embryos. Some atypical electron-dense structures and cellular alterations were noticed in the brain but independent from graphene concentration. According to studies using the zebrafish model described above Chen et al. (2016), results indicate some harmful effects of GDMs in embryogenesis that should be more deeply investigated before advancing on their biomedical application. 


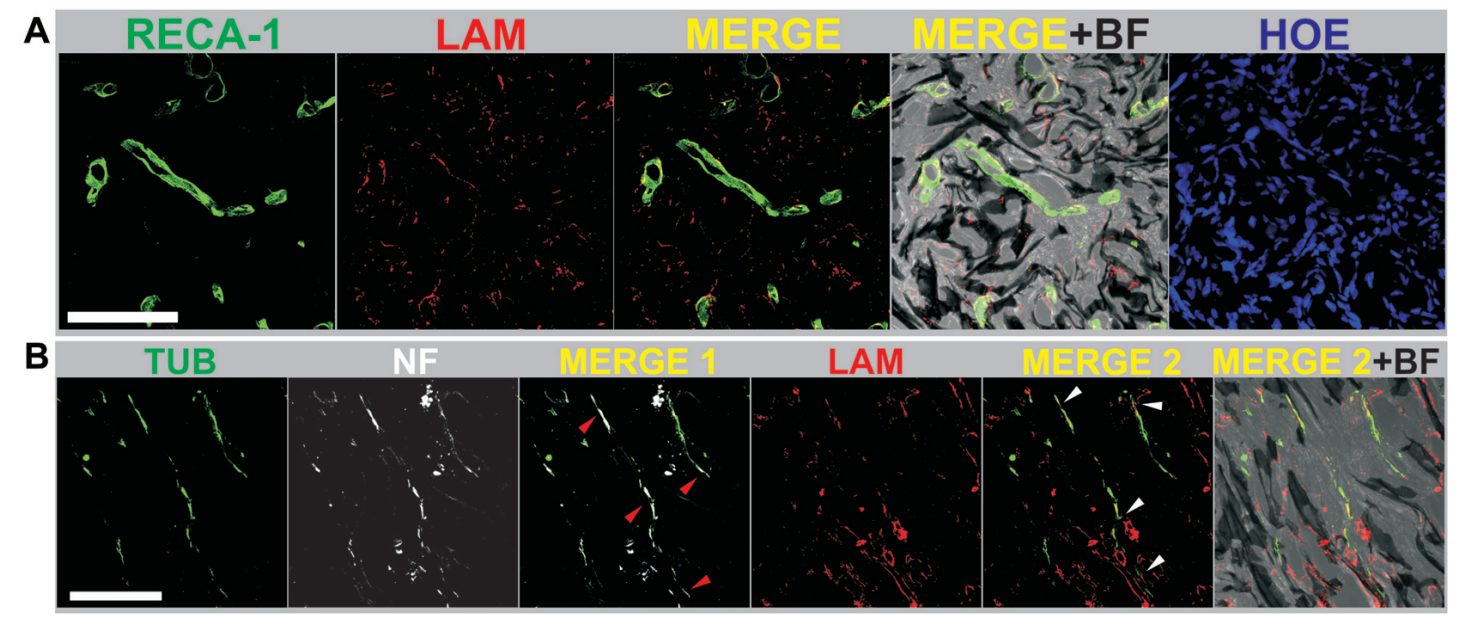

FIGURE 1 | (A) Mature blood vessels were detected inside reduced graphene oxide (RGO) scaffolds at 30 days post-injury by labeling RECA-1 (green) and laminin from basal membranes (LAM, red). (B) Axons were specifically detected inside RGO scaffolds at 30 days post-injury by expression of $\beta$-III tubulin (TUB, green) and neurofilaments (NF, white). Laminin from basal membranes was labeled in red (LAM). Arrow heads indicate areas with coexistence of TUB and NF (red; MERGE 1) and TUB with LAM (white; MERGE 2). Cell nuclei were labeled with Hoechst (HOE). Bright field images (BF) are included to visualize the scaffold structure. Scale bar: $100 \mu \mathrm{m}$. Adapted from López-Dolado et al. (2016), Copyright (2016), with permission from Elsevier.

\section{GRAPHENE-DERIVED MATERIALS INTERFACING THE SPINAL CORD EX VIVO AND IN VITRO}

Other outstanding studies in the field used spinal explants as an alternative to in vivo murine models. Usmani et al. (2016) pioneered this work by using spinal organotypic slices obtained from spinal cords of E12 C57BL mouse embryos for more than 2 weeks in culture. Particularly, co-cultures of pairs of spinal cord slices separated by a known distance were carried out. The selected materials were 3D scaffolds composed of multi-walled carbon nanotubes (MWCNTs), a different type of carbon-based nanomaterial consisting of concentric layers of graphene rolled up in multi-layered cylinders. A significant development of neuronal projections was found, but with a completely different organization, in both control slices and those interfaced by MWCNT scaffolds. Specifically, the supporting role played by the scaffold made neurites to grow from the explants into a complex net of randomly oriented processes populating the third dimension of the nanoscaffold, whereas they were organized into thick bundles of aligned fibers in controls. Functional tests weakening synaptic inhibition demonstrated slow-paced bursting in all co-cultured explants, with similar inter-event interval in controls and MWCNT scaffolds. However, only a minority of control explants had successful reconnection despite the high number of fiber bundles grown. On the contrary, in the MWCNT scaffold group, a 94\% of cases had correlated activity of disinhibited bursting between co-cultured explants. Interestingly, these results could not be reproduced when using a $3 \mathrm{D}$ polydimethylsiloxane substrate (although tridimensional, it did not allow the electrical reconnection between explants). Additionally, 3D MWCNT scaffolds did not generate exaggerated tissue responses when implanted in the visual cortex of male rats, as proved by the analysis of the glial scar 4 weeks after surgery and Iba1 levels over time. Finally, the presence of neural cells within the scaffold confirmed that this material could not only integrate into the neural tissue, but also prompt the migration of neuroblasts (localized nearby the implant) to the injury site.

As the interaction between nanomaterials and cells could drive to intracellular changes due to their association with cell membranes, Rauti et al. (2016) investigated whether the exposure to 2D GO nanosheets could alter cell membrane-based processes, such as vesicle kinetics, and therefore compromise the synaptic communication in both primary hippocampal neurons and cortical glia cultures. Two different kinds of GO nanosheets were used only differing in their lateral dimensions: large and small (l-GO and s-GO, respectively). Interestingly, larger lateral protrusions were more cytotoxic than s-GO, then excluding l-GO samples for the rest of the studies. When focused on s-GO $(10 \mu \mathrm{g} / \mathrm{mL})$, results revealed significant reduction of spontaneous postsynaptic currents (in frequency, not in amplitude). Longer inter-event intervals in $\mathrm{Ca}^{2+}$ oscillations and a decrease in vesicular glutamate transporter 1 (VGLUT1) levels were also detected, among other major findings, thus demonstrating interference with neuronal synapses functioning (Figure 2). Importantly, these results were not related to any alteration in cell viability and were not found in cultures exposed to graphene nanosheets.

Pioneer work by Fabbro et al. (2013) explored the use of spinal neurons for evaluating materials aiding to repair lesions at the spinal cord. In these studies, authors tested 2D scaffolds composed of CNTs with dissociated neuronal cultures from neonatal rat spinal cords (P1-P4). Interestingly, the contact 

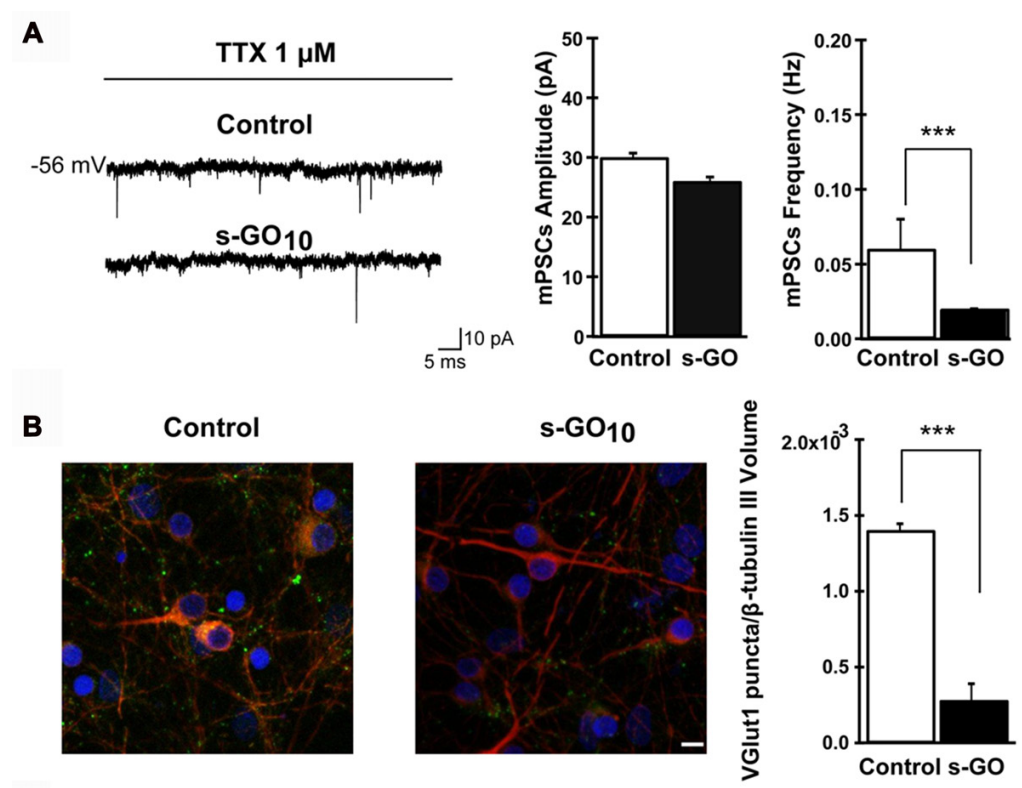

C

Control
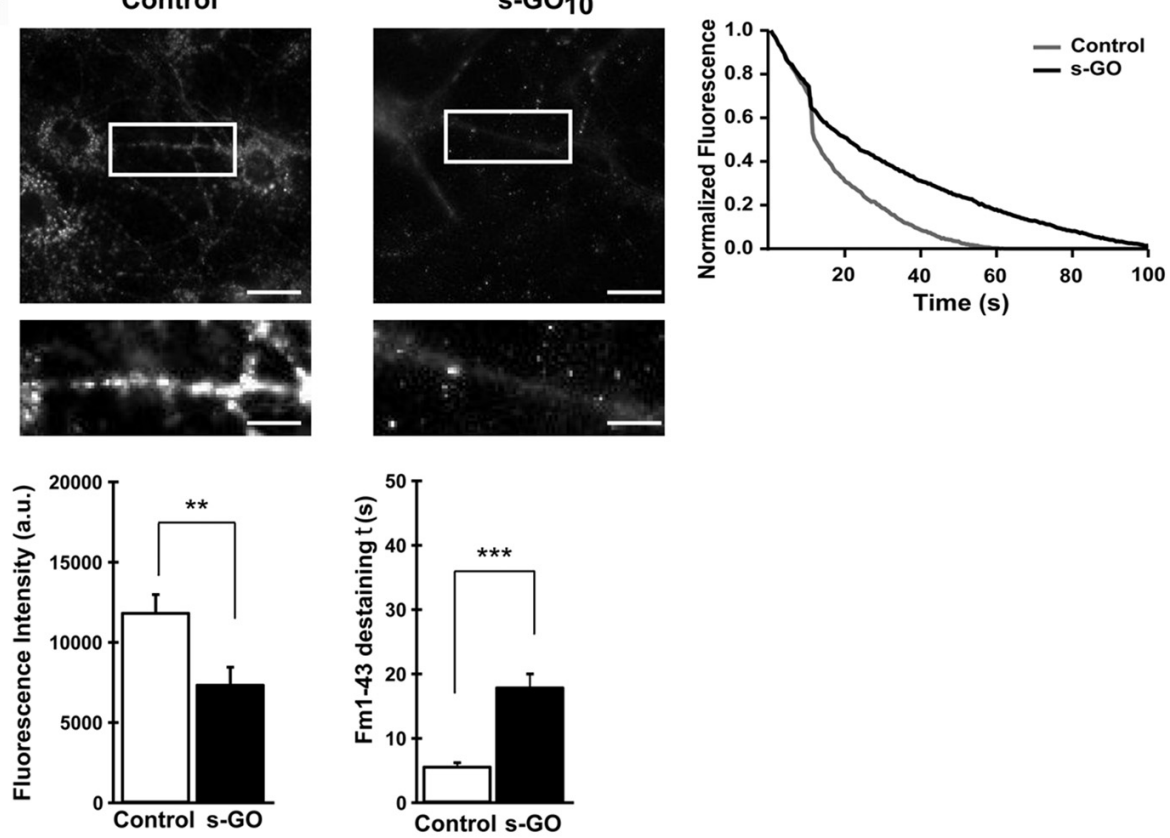

FIGURE 2 | Exposure to small graphene oxide nanosheets (s-GO) at high concentration impaired excitatory synapses. In (A), sample tracings of mPSCs recorded in control and s-GO-treated cultures (left panel) and plots reporting mPSC amplitude and frequency values (right panel) $(* * * p<0.001$, Student's-t-test). In (B), confocal reconstruction of control and s-GO-treated neurons immunolabeled for the vesicular glutamate transporter 1 (VGLUT1; green) and counterstained for cytoskeletal component $\beta$ tubulin III (red; nuclei are visualized by DAPI labeling in blue; scale bar $10 \mu \mathrm{m}){ }^{* * *} p<0.001$, Student's-t-test). In (C), fluorescence images following staining with FM1-43, control and s-GO-treated (scale bar: $50 \mu \mathrm{m}$ ) (top). The areas in the boxes are higher magnifications to highlight the difference in vesicular staining between the two conditions (scale bar: $10 \mu \mathrm{m}$ ). The plot (top right) reproduces the representative (control and s-GO) traces of FM1-43 de-staining (each trace has been normalized to the maximum fluorescence detected). Bottom: the left plot summarizes the initial raw fluorescent intensities of hippocampal terminals from control and s-GO-treated cultures $(* * p<0.01$, Mann-Whitney test); the right plot summarizes the decay time constant $\tau$ of FM1-43 de-staining in the two conditions $(* * * p<0.001$, Mann-Whitney test). Reprinted with permission from Rauti et al. (2016). Copyright (2016) American Chemical Society.

with these substrates modulated diverse intracelullar processes including gene translation and transcription. Importantly, neuronal maturation was anticipated by modifying neural ability to generate action potentials, among others. For instance, Alox15 gene expression and its product (related to axon pathfinding) presented higher levels in neurons exposed to CNTs. Nonetheless, authors underlined that a relationship between the cell-material interaction and 
changes in gene expression patterns could not be directly established.

\section{FINAL CONCLUSIONS}

Thanks to research efforts, knowledge on the interaction of GDMs with central neural cells and tissues is increasing. Extensive results from in vitro studies have outlined the relevance that the physic-chemical properties of GDMs play in cell behavior. Some toxicity concerns about their use and the critical influence of particular functionalizations have been also evidenced. In this sense, cellular aspects as diverse as differentiation, neurite outgrowth, oxidative stress and protein and gene expressions could be significantly modulated. The findings from in vivo work revealed the ability of GDMs such as graphene, rGO and MWCNTs shaped as $3 \mathrm{D}$ scaffolds to initiate beneficial tissue responses when implanted in the injured spinal cord and the brain. Angiogenesis, axon growth, immunomodulatory effects, maintenance of neurogenesis and diminished fibroglial scar formation are some of the most relevant pro-regenerative responses induced by these materials. Ex vivo models with spinal cord slices are presented as an attractive and alternative system for testing the phenomena taking place when interfacing central neural tissues with GDMs. Procedures used for testing GDMs in contact with neural tissues

\section{REFERENCES}

Capaday, C. (2002). The special nature of human walking and its neural control. Trends Neurosci. 25, 370-376. doi: 10.1016/s0166-2236(02)02173-2

Chen, R., Cohen, L. G., and Hallett, M. (2002). Nervous system reorganization following injury. Neuroscience 111, 761-773. doi: 10.1016/s0306-4522(02) 00025-8

Chen, Y., Hu, X., Sun, J., and Zhou, Q. (2016). Specific nanotoxicity of graphene oxide during zebrafish embryogenesis. Nanotoxicology 10, 42-52. doi: 10.3109/17435390.2015.1005032

Defterali, Ç., Verdejo, R., Peponi, L., Martín, E. D., Martínez-Murillo, R., LópezManchado, M. A., et al. (2016). Thermally reduced graphene is a permissive material for neurons and astrocytes and de novo neurogenesis in the adult olfactory bulb in vivo. Biomaterials 82, 84-93. doi: 10.1016/j.biomaterials.2015. 12.010

Fabbro, A., Sucapane, A., Toma, F. M., Calura, E., Rizzetto, L., Carrieri, C., et al. (2013). Adhesion to carbon nanotube conductive scaffolds forces actionpotential appearance in immature rat spinal neurons. PLoS One 8:e73621. doi: 10.1371/journal.pone.0073621

Fattahi, P., Yang, G., Kim, G., and Abidian, M. R. (2014). A review of organic and inorganic biomaterials for neural interfaces. Adv. Mater. 26, 1846-1885. doi: 10.1002/adma.201304496

Fawcett, J. W., and Asher, R. A. (1999). The glial scar and central nervous system repair. Brain Res. Bull. 49, 377-391. doi: 10.1016/s0361-9230(99) 00072-6

Fawcett, J. W., Schwab, M. E., Montani, L., Brazda, N., and Müller, H. W. (2012). Defeating inhibition of regeneration by scar and myelin components. Handb. Clin. Neurol. 109, 503-522. doi: 10.1016/B978-0-444-52137-8.00031-0

Figley, S. A., Khosravi, R., Legasto, J. M., Tseng, Y. F., and Fehlings, M. G. (2014). Characterization of vascular disruption and blood-spinal cord barrier permeability following traumatic spinal cord injury. J. Neurotrauma 31, 541-552. doi: 10.1089/neu.2013.3034

Fraczek-Szczypta, A. (2014). Carbon nanomaterials for nerve tissue stimulation and regeneration. Mater. Sci. Eng. C Mater. Biol. Appl. 34, 35-49. doi: 10.1016/j. msec.2013.09.038

Garstang, S. V., and Walker, H. (2011). "Cardiovascular and autonomic disfunction after spinal cord injury," in Spinal Cord Medicine, 2nd Edn. and cells should be standardized to diminish contradictory results and facilitate comparisons among laboratories working in the field.

In conclusion, recent advances such as those described in this mini-review are boosting multidisciplinary research in the field that will likely open the path to the exploration of GDMs in translational approaches in biomedicine. Diverse pathologies at the central nervous system including SCI and some brain disorders could eventually benefit from the promising properties of these materials when interfacing the damaged neural tissue. In the next decade, we might assist to a new generation of advanced biomaterials based on graphene with unprecedented modulatory properties in neural systems.

\section{AUTHOR CONTRIBUTIONS}

All authors equally contributed to the elaboration of this manuscript.

\section{ACKNOWLEDGMENTS}

This project has received funding from the Agencia Estatal de Investigación of Spain and the Fondo Europeo de Desarrollo Regional (MAT2016-78857-R, AEI/FEDER, UE).

eds D. I. Campagnolo, S. Kirshblum and J. A. DeLisa (Philadelphia, PA: Lippincott Williams and Willkins), 136-154.

Göritz, C., Dias, D. O., Tomilin, N., Barbacid, M., Shupliakov, O., and Frisén, J. (2011). A pericyte origin of spinal cord scar tissue. Science 333, 238-242. doi: $10.1126 /$ science. 1203165

Harkema, S. J. (2008). Plasticity of interneuronal networks of the functionally isolated human spinal cord. Brain Res. Rev. 57, 255-264. doi: 10.1016/j. brainresrev.2007.07.012

John, A. A., Subramanian, A. P., Vellayappan, M. V., Balaji, A., Mohandas, H., and Jaqanathan, S. K. (2015). Carbon nanotubes and graphene as emerging candidates in neuroregeneration and neurodrug delivery. Int. J. Nanomedicine 10, 4267-4277. doi: 10.2147/IJN.S83777

Kigerl, K. A., Gensel, J. C., Ankeny, D. P., Alexander, J. K., Donnelly, D. J., and Popovich, P. G. (2009). Identification of two distinct macrophage subsets with divergent effects causing either neurotoxicity or regeneration in the injured mouse spinal cord. J. Neurosci. 29, 13435-13444. doi: 10.1523/JNEUROSCI. 3257-09.2009

Kigerl, K. A., Hall, J. C., Wang, L., Mo, X., Yu, Z., and Popovich, P. G. (2016). Gut dysbiosis impairs recovery after spinal cord injury. J. Exp. Med. 213, 2603-2630. doi: $10.1084 /$ jem.20151345

Kokaia, Z., Martino, G., Schwartz, M., and Lindvall, O. (2012). Cross-talk between neural stem cells and immune cells: the key to better brain repair? Nat. Neurosci. 15, 1078-1087. doi: 10.1038/nn.3163

Lee, C., Wei, X., Kysar, J. W., and Hone, J. (2008). Measurement of the elastic properties and intrinsic strength of monolayer graphene. Science 321, 385-388. doi: $10.1126 /$ science. 1157996

Li, D., Müller, M. B., Gilje, S., Kaner, R. B., and Wallace, G. G. (2008). Processable aqueous dispersions of graphene nanosheets. Nat. Nanotechnol. 3, 101-105. doi: 10.1038/nnano.2007.451

López-Dolado, E., González-Mayorga, A., Gutiérrez, M. C., and Serrano, M. C. (2016). Immunomodulatory and angiogenic responses induced by graphene oxide scaffolds in chronic spinal hemisected rats. Biomaterials 99, 72-81. doi: 10.1016/j.biomaterials.2016.05.012

López-Dolado, E., González-Mayorga, A., Portolés, M. T., Feito, M. J., Ferrer, M. L., del Monte, F., et al. (2015). Subacute tissue response to 3D graphene oxide scaffolds implantes in the injured rat spinal cord. Adv. Healthc. Mater. 4, 1861-1868. doi: 10.1002/adhm.201500333 
Manzo, L. P., Ceragioli, H., Bonet, I. J., Nishijima, C. M., Vieira, W. F., Oliviera, E. C., et al. (2017). Magnetic, but non-magnetic, reduced graphene oxide in spinal cord increases nociceptive neuronal responsiveness. Nanomedicine 13, 1841-1851. doi: 10.1016/j.nano.2017. 02.019

Mendonça, M. C., Soares, E. S., de Jesus, M. B., Ceragioli, H. J., Batista, Â. G., NyúlTóth, Á., et al. (2016). PEGylation of reduced graphene oxide induces toxicity in cells of the blood-brain barrier: an in vitro and in vivo study. Mol. Pharm. 13, 3913-3924. doi: 10.1021/acs.molpharmaceut.6b00696

Nakanishi, W., Minami, K., Shrestha, L. K., Ji, Q., Hill, J. P., and Ariga, K. (2014). Bioactive nanocarbon assemblies: nanoarchitectonics and applications. Nano Today 9, 378-394. doi: 10.1016/j.nantod.2014.05.002

Rauti, R., Lozano, N., León, V., Scaini, D., Musto, M., Rago, I., et al. (2016). Graphene oxide nanosheets reshape synaptic function in cultured brain networks. ACS Nano 10, 4459-4471. doi: 10.1021/acsnano.6b00130

Rodriguez, J. P., Coulter, M., Miotke, J., Meyer, R. L., Takemaru, K., and Levine, J. M. (2014). Abrogation of $\beta$-catenin signaling in oligodendrocyte precursor cells reduces glial scarring and promotes axon regeneration after CNS injury. J. Neurosci. 34, 10285-10297. doi: 10.1523/JNEUROSCI.491513.2014

Sapru, H. N. (2011). "Spinal Cord: anatomy, physiology, and pathophysiology," in Spinal Cord Medicine, 2nd Edn. eds D. I. Campagnolo, S. Kirshblum and J. A. DeLisa (Philadelphia, PA: Lippincott Williams and Willkins), 6-30.

Sawosz, E., Jaworski, S., Kutwin, M., Hotowy, A., Wierzbicki, M., Grodzik, M., et al. (2014). Toxicity of pristine graphene in experiments in a chicken embryo model. Int. J. Nanomedicine 9, 3913-3922. doi: 10.2147/IJN. S65633

Soldano, C., Mahmood, A., and Dujardin, E. (2010). Production, properties and potential of graphene. Carbon 48, 2127-2150. doi: 10.1016/j.carbon.201001.058
Spinal Cord Injury (SCI). (2016). Facts and figures at a glance. J. Spinal Cord Med. 39, 493-494. doi: 10.1080/10790268.2016.1210925

Tavangarian, F., and Li, Y. (2012). Carbon nanostructures as nerve scaffolds for repairing large gaps in severed nerves. Ceramics Int. 38, 6075-6090. doi: 10.1016/j.ceramint.2012.05.038

Usmani, S., Aurand, E. R., Medelin, M., Fabbro, A., Scaini, D., Laishram, J., et al. (2016). 3D meshes of carbon nanotubes guide functional reconnection of segregated spinal explants. Sci. Adv. 2:e1600087. doi: 10.1126/sciadv. 1600087

Yang, L., Wang, F., Han, H., Yang, L., Zhang, G., and Fan, Z. (2015). Functionalized graphene oxide as a drug carrier for loading pirfenidone in treatment of subarachnoid hemorrhage. Colloids Surf. B Biointerfaces 129, 21-29. doi: 10.1016/j.colsurfb.2015.03.022

Yang, X., Zhang, X., Liu, Z., Ma, Y., Huang, Y., and Chen, Y. (2008). Highefficiency loading and controlled release of doxorubicin hydrochloride on graphene oxide. J. Phys. Chem. C 112, 17554-17558. doi: 10.1021/jp80 $6751 \mathrm{k}$

Conflict of Interest Statement: The authors declare that the research was conducted in the absence of any commercial or financial relationships that could be construed as a potential conflict of interest.

Copyright (c) 2017 Domínguez-Bajo, González-Mayorga, López-Dolado and Serrano. This is an open-access article distributed under the terms of the Creative Commons Attribution License (CC BY). The use, distribution or reproduction in other forums is permitted, provided the original author(s) or licensor are credited and that the original publication in this journal is cited, in accordance with accepted academic practice. No use, distribution or reproduction is permitted which does not comply with these terms. 